\title{
Local One-Sided Rubber Bulging Test to Measure Various Strain Paths of Metal Tube
}

\author{
Hidenori Yoshimura ${ }^{1, *}$, Kana Nakahara ${ }^{2}$ and Masaaki Otsu ${ }^{3}$ \\ 1 Faculty of Engineering and Design, Kagawa University, Takamatsu 761-0396, Japan \\ 2 Graduate School of Engineering, Kagawa University, Takamatsu 761-0396, Japan; s19g522@stu.kagawa-u.ac.jp \\ 3 Faculty of Engineering, University of Fukui, Fukui 910-8507, Japan; otsu@u-fukui.ac.jp \\ * Correspondence: yoshimura.hidenori@kagawa-u.ac.jp; Tel.: +81-87-864-2345
}

Citation: Yoshimura, H.; Nakahara, K.; Otsu, M. Local One-Sided Rubber Bulging Test to Measure Various Strain Paths of Metal Tube. Metals 2021, 11, 751. https://doi.org/ $10.3390 /$ met11050751

Academic Editor: Umberto Prisco

Received: 25 March 2021

Accepted: 30 April 2021

Published: 2 May 2021

Publisher's Note: MDPI stays neutral with regard to jurisdictional claims in published maps and institutional affiliations.

Copyright: (c) 2021 by the authors. Licensee MDPI, Basel, Switzerland. This article is an open access article distributed under the terms and conditions of the Creative Commons Attribution (CC BY) license (https:// creativecommons.org/licenses/by/ $4.0 /)$.

\begin{abstract}
We proposed a local one-sided rubber bulging method of metal tubes to evaluate various strain paths at an aimed portion and measured the forming limit strains of metal tubes at the place of the occurrence of necking under biaxial deformation. Using this method, since rubber is used to give pressure from the inner side of the tube, no sealing mechanisms were necessary unlike during hydraulic pressure bulging. An opening was prepared in front of the die to locally bulge a tube at only the evaluation portion. To change the restriction conditions of the bulged region for biaxial deformation at the opening, a round or square cutout, or a slit was introduced. The test was conducted using a universal compression test machine and simple dies rather than a dedicated machine. Considering the experimental results, it was confirmed that the strain path was varied by changing the position and size of slits and cutouts. Using either a cutout or a slit, the strain path in the side of the metal tubes can be either equi-biaxial tension or simple tension, respectively. Additionally, by changing the size of the cuts or slits, the strain path can be varied.
\end{abstract}

Keywords: tube forming; tube bulging test; formability test; forming limit; biaxial strain; local rubber bulging; cutout shape; slit length

\section{Introduction}

Recently, the application of tube-formed products has been utilized in various hollow transport components and mechanical structures to improve their rigidity and strength and to reduce their production cost. When the cross-sectional areas of solid and hollow materials are the same, the moment of inertia of the hollow-like tube is larger than that of the solid. Regarding the case of producing hollow parts, a welding margin is necessary when forming from sheet metal but unnecessary from a tube. Thus, the mass of the parts can be reduced, and the specific rigidity and specific strength will increase. Regarding the latter, because some workings such as bulging and piercing can be performed simultaneously by a tube-forming machine, production costs can be reduced. Concerning tube forming, tube materials are subjected to complex and severe deformation and, thus, some forming defects such as cracking and buckling often occur. To avoid such forming defects, the formability of the tube materials should be evaluated appropriately. Although trial forming tests require both time and cost, finite element method (FEM) simulation can reduce both of them. To obtain satisfactory simulated results during the forming processes, trustworthy input data should be obtained by accurate measurement of the material properties of the flow stress, the forming limit, and the fracture limit. Since tubes have distributions of initial thickness and hardness in the circumferential direction that is caused by the manufacturing method, input data for the simulations should be obtained, but not from sheet metal before forming into a tube, rather from a tube [1,2]. Metal tubes tend to have more anisotropy of strength because they are made from pre-finished products by the additive working process. The purpose of this research is to establish an easy biaxial test method for metal tubes to enable the construction of a forming limit diagram. 
Yoshida has reported on the examination of standard test methods such as Japanese Industrial Standards (JIS) and International Organization for Standardization (ISO) and other methods [3]. Generally, mechanical properties tests for tubes are classified into axial and circumferential directions. There are JIS Z 2241 in the axial tensile test with which it is possible to obtain the ultimate tensile strength, yield point, breaking elongation, etc. Conversely, regarding the circumferential direction, the ring tension (ISO 8496), flatness (ISO 8492), push-spread (ISO 8493), flange extension (ISO 8494), ring spread (ISO 8495) tests, etc. are standardized. However, the purpose of them is to detect defects in the tube material such as a defective weld. Moreover, they are only soundness evaluation tests of the pre-service tube and cannot evaluate tube formability.

Therefore, various advanced researches have been conducted on the evaluation test of the tube formability. Regarding the axial direction, Yoshida et al. developed the tube tensile test using image processing for measurement of the flow stress and forming limit. Also, the influence of triaxiality on the fractured strain change around the weld seam [4,5] was investigated. Concerning the circumferential direction, Yoshimura et al. reported the ring tensile test method which can evaluate the flow stress and forming limit strain of the welded tube's base metal [6,7]. Sokolowski, Muammer, and Hwang proposed methods to evaluate flow stress using a free bulging test which does not need to cut a specimen from the tube [8-10]. Additionally, Manabe et al. proposed the conical flaring test in which the tube was expanded until cracks occurred at the end of the tube, and also investigated the effects of test conditions, mechanical properties of the material, and the apex angle of the conical tool on the deformation behavior [11,12]. Also, using a conical flaring test, they identified the parameters of the ductile fracture criterion proposed by Oyane [13]. Manabe and Yoshida compared a conical flaring test to three kinds of bulging test and reported that the flaring test results are in relatively good agreement with the result of the rubber bulging test [14].

It is well-known that the ratios of the circumferential strain and axial strain influence the forming limit of the tube similar to the sheet forming. Therefore, several evaluation methods for the forming limit on the biaxial deformation of the tube have been proposed. Most of them are methods which apply the free bulge test. Guo et al. improved the accuracy of the evaluation of the forming limit of the tube under hydraulic free bulging without axial feeding using a combination of Oyane's ductile fracture criterion with the M-K theory [15].

Generally, free-bulging tests with internal pressure and axial feeding are classified into two kinds, depending on axial feeding; one has only compressive axial feeding, the other has compressive and tensile axial feeding. Many investigations are concerned with the former to obtain only the left-hand side of the forming limit diagram (FLD). Hwang et al. constructed a FLD of AA6011 using a free bulge test machine which clamps and pushes both ends of the tube [16], for example. Kim proposed analytical and numerical analyses of the forming limit in the free bulge test with axial compressive feeding, and it was shown that the results agreed with the experimental results [17]. Song et al. also executed a series of free bulge tests on an FLD of a tube with a similar experimental apparatus and investigated the effect of the flow stress on the FLD [18]. Kuwabara et al. conducted simple hydraulic bulging tests in which ends of the tube were not restricted and examined the breakage expansion ratio and the forming limit of the circumferential strain. Moreover, they applied the tests on three types of tubes(1)as-roll, (2)as-roll and normalized, and (3)cold-drawn and normalized, and reported in detail about the relation of uneven initial tube thickness distribution and uneven hardness distribution to the fracture position [19].

To obtain the strain paths, including the right side of the FLD by the free bulge test, it is necessary to apply axial tension to both ends of the pipe. The difficulty is in clamping both ends of the tube without leakage of the internal pressure medium. Kuwabara et al. developed a hydraulic multiaxial tube expansion testing machine that can measure material properties along a predetermined strain and stress path by controlling the axial load and the internal hydraulic pressure, and can evaluate the mechanical properties of tubes and plates, yield surface, and so on, until large strains cause failure [20]. During this current research, 
a test method for obtaining the forming limit stress curve (FLSC) has been proposed. During the subsequent research of the former study, Kuwabara et al. developed the test method which enables continuous measurement of large-strain biaxial stress-strain curves without resticking strain gages [21]. Korkolis and Kyriakides investigated the inflation and burst of Al-6260-T4 tubes under combined tensile or compressive axial loads with internal pressure and observed that localized wall thinning, and burst can be very sensitive to the constitutive description employed for the material [22].

Added to the free bulge test, by controlling the internal pressure and axial feeding, other kinds of material property evaluation tests also have been proposed. As a test method designating the evaluated area in a tube, Shirayori et al. proposed a partial hydraulic bulge test method in which only a designated part of a tube was expanded [23] and described the deformation behavior on the wall thickness and the bulged rate under multiple axial loading conditions [24]. Chen et al. proposed a new theoretical model to predict the FLD for a seamed tube. Regarding the same report, they obtained the left-hand side FLD using a classical free hydroforming tool set, and the right-hand side FLD via the novel device to simultaneously give the lateral compression force and the internal pressure to control the material flow [25]. Furthermore, Lin et al. proposed a novel experimental device by partially bulging a tube at the upper elliptical opening to evaluate the right side FLD and could obtain the forming limit strains at the weld line and the opposite region of the tube [26]. Magrinho et al. obtained multiple strain paths in both sides of the FLD using three kinds of tests: a uniaxial tensile test of a specimen cutout from a tube, a tube expansion test of the tube end with a rigid punch, and a rubber pressured free bulge test using a digital image correlation system (DIC) [27]. Furthermore, Magrinho et al. also proposed a methodology to determine the forming limits by buckling, necking, and fracture during an external thin-walled tube inversion [28].

Since welded steel tubes, which are frequently used for parts of automobiles, have a different hardness between the weld and the base metal caused by the thermal treatment, it is necessary to evaluate the weld and the base metal, respectively. However, there is no test of the tube to evaluate a designated location, except those by Shirayori et al. [23] and Lin et al. [26]. Most biaxial tube formability evaluation tests require expensive special equipment for hydraulic loading, sealing, and axial loading, excluding a conical flaring test and rubber bulge test. Furthermore, it is important to check the linearity of the strain path because the forming limit curve (FLC) is changed depending on the strain path. Chen et al. and Lin et al. measured strain paths using each test method $[25,26]$. However, these methods have problems caused by stopping the test, such as the effect of unloading, the length of the test time, and damage to the specimen. Therefore, a non-contact strain measurement method is required as a method that can be continuously and quickly tested and measured with a single test tube.

During this research, to obtain a forming limit under various biaxial deformations of a tube, we propose a local one-sided rubber bulging test. Using this test method, a tube is locally bulged to specify the evaluation part, for example, the base material except for the weld and heat affected zone (HAZ). Since the pressure medium is rubber, the test machine is inexpensive. Although the final goal of this research is measuring the FLD of the tube, this report focuses on the possibility of measuring various proportional strain paths in the right and left sides of the strain space. To realize the various strain paths, we propose that a cutout or slit is preprocessed on the tube specimen. It is possible for noncontact while continually measuring the strain path using image processing because the solid rubber pressure medium has little risk to damage the camera. Thus, the test can be simply and continuously conducted until the fracture limit. This test method was applied for a pure aluminum tube (JIS A 1070TD), and we confirmed the linearity of the obtained strain path and the wide range of the strain ratio of the strain paths. 


\section{Materials and Methods}

\subsection{Overview of the Local Rubber Bulging Test}

During the proposed local one-sided rubber bulging test shown in Figure 1, the outer side of a tube is restrained with a die (a container), and silicone rubber that is inserted inside the tube is compressed axially by a punch to provide the tube internal pressure. Only the local surface at the opening of the die is bulged and strain on the bulged surface is measured. Silicone rubber can be compressed easily with a punch using a universal testing machine. Regarding the case of hydraulic pressure, the pressure medium leaks easily, despite the small expansion of the tube diameter in the clearance between the tube and the die. Conversely, in the proposed method, silicone rubber can be easily sealed using urethane rubber, which is harder than silicone rubber. Thus, complicated and any expensive equipment, including a hydraulic pressure pump and sealing mechanisms, are unnecessary in this method. The circumferential and axial strains at the top of the bulged surface, which is the center of the die's opening, are measured using an image processing system.

Multiple strain paths are required to construct the FLD of the tube material. Magrinho et al. have reported that the free rubber pressure-bulging tests in which the movement of the tube ends is fixed can obtain multiple strain paths only on the right side of the strain space (from plane strain tension to equi-biaxial tension) by changing the bulged length [25]. Using the proposed test method, it is expected to measure not only the right-side strain paths but also the left-hand strain paths. Various deformations can be obtained by making a cutout or slits on the tube specimens. Figure 2 shows three kinds of specimens before and after the test: no cutout, cutout, and slit. No-cutout is an unprocessed tubular specimen, and the strain path on the blue line of the plane strain tension will be measured. Cutout is a preprocessed tubular specimen cutout on the opposite side to the bulged surface, and strain paths in the right hand of the No-cutout will be measured in the green space shown in Figure 2. A slit is added to a tubular specimen on the same side as the bulging, and the strain paths in the left hand of the strain paths of the No-cutout will be measured in the yellow space in Figure 2.

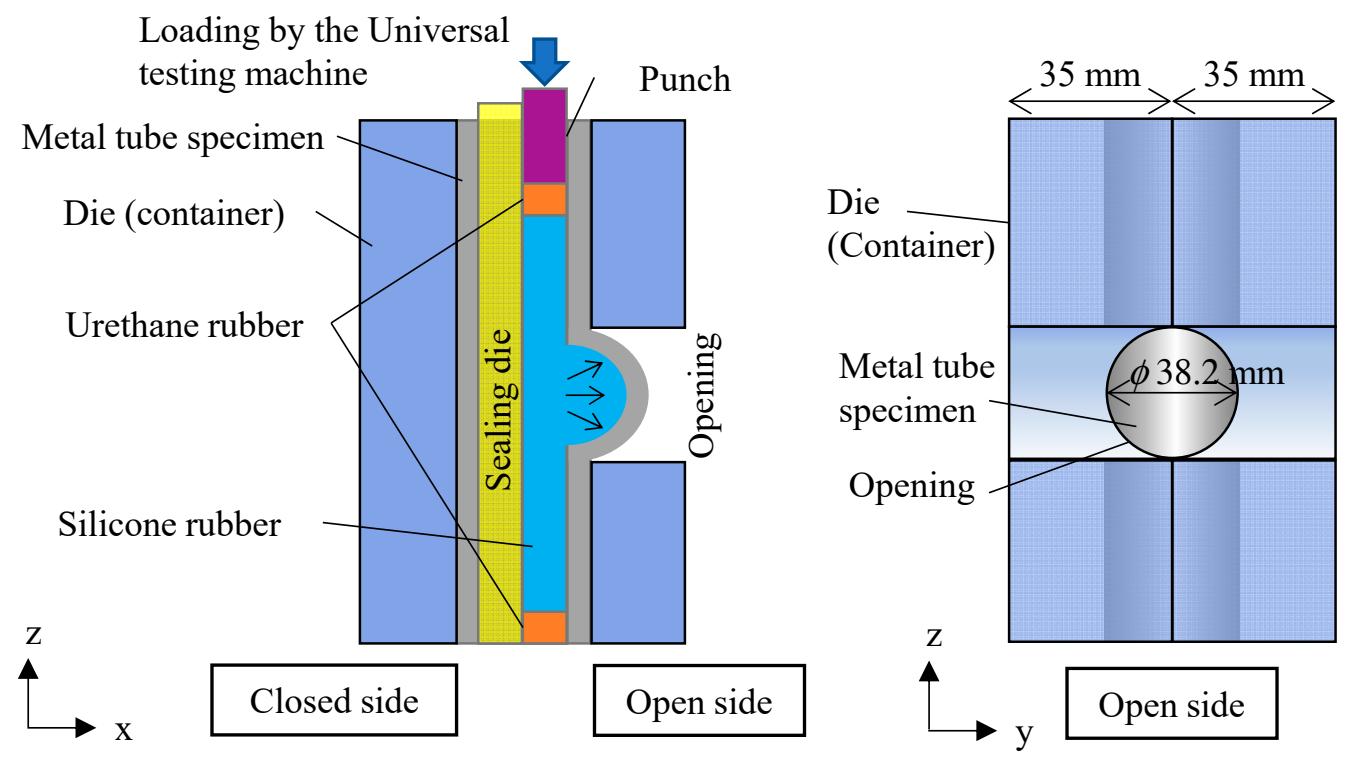

(a)

Figure 1. Cont. 


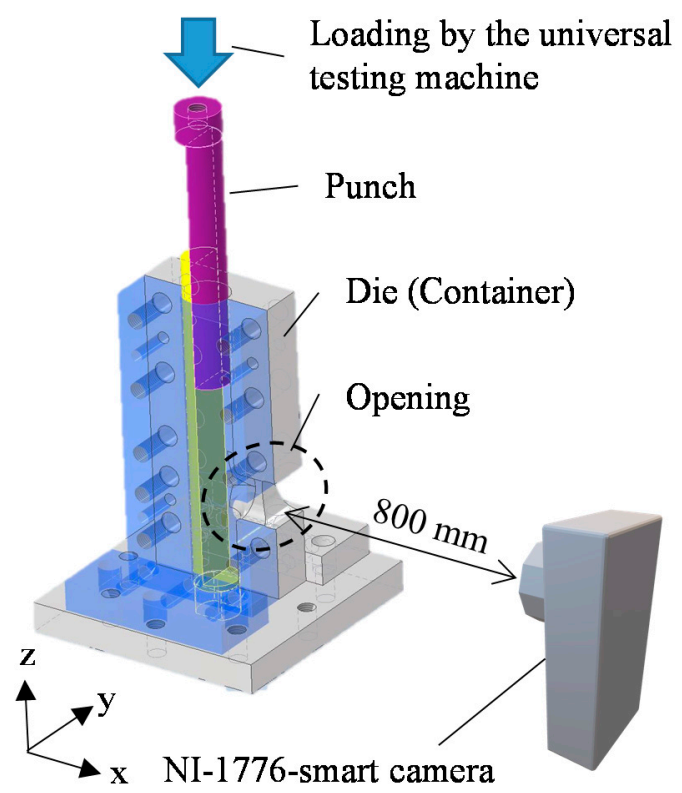

(b)

Figure 1. Illustration of local one-sided rubber bulging test: (a) Cross-sectional view; (b) General view.

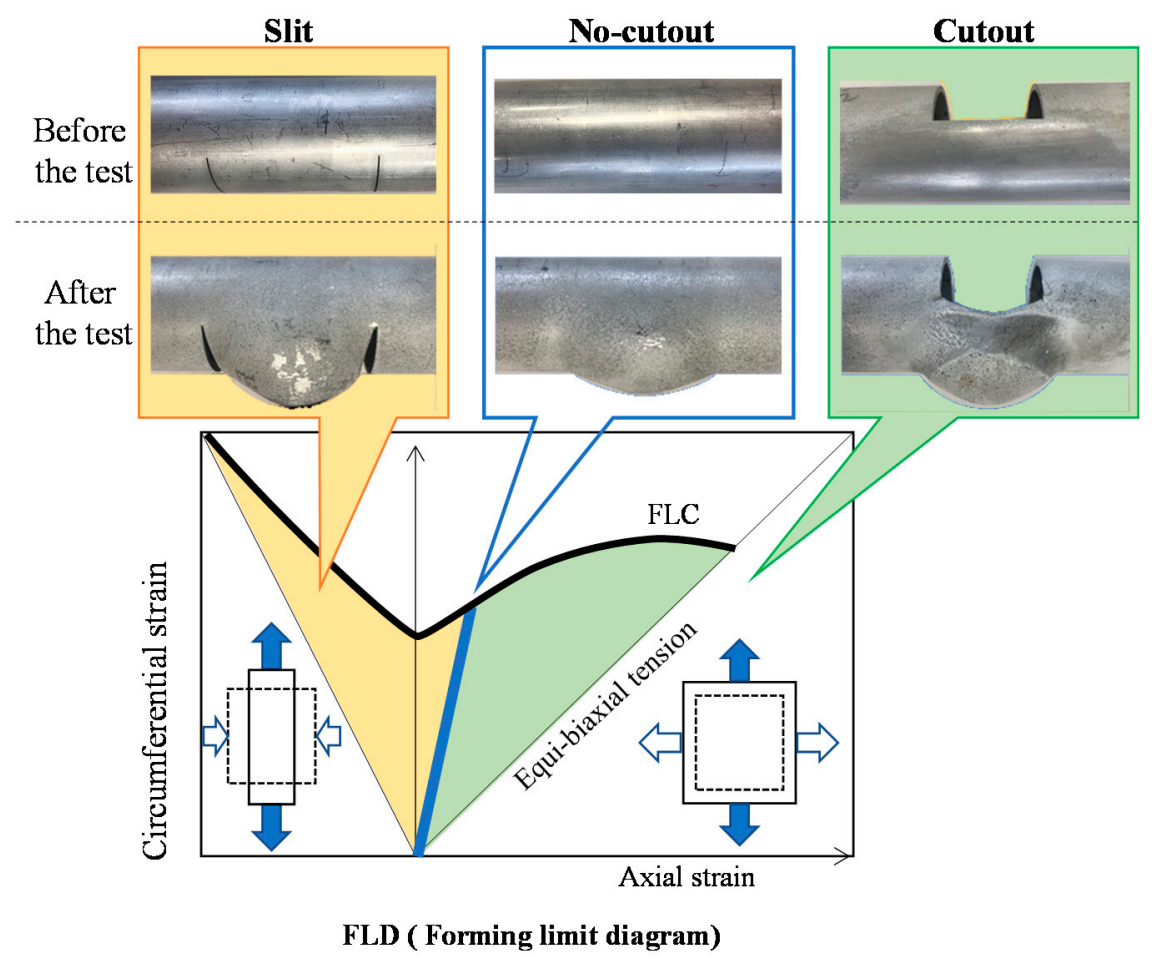

Figure 2. Change of strain path by introducing a cutout or slits to the tube specimen.

During this test method, since a local bulged region can be selected, the bulged area is set to exclude a singular region, for example, the welded or heat-affected zone of the welded steel tube. Besides the welded tube, the other metal tubes also have thickness and hardness distributions in the circumferential direction due to the manufacturing process. Although the weak point, which is the thinner or softer region, may be broken, generally such weak points cannot be avoided when designing hollow products. Therefore, in this paper, it is explained how to know beforehand where the weak position of a metal tube is using preliminary tests and then by measuring the strain path around the position. 


\subsection{Specimen, Rubber Pressure Medium and Test Equipment}

The proposed test method was applied to the round pure aluminum tube, JIS: A1070TD, with an outer diameter $D_{0}=38.0 \mathrm{~mm}$, and a nominal thickness $t=2.0 \mathrm{~mm}$. Since this pure aluminum tube was made using porthole extrusion, it did not have the heat affect caused by welding. Also, the initial thickness distribution was less than $\pm 0.035 \mathrm{~mm}$ and seemed very small. Prior to the test, the specimens used were annealed for $1 \mathrm{~h}$ at $400{ }^{\circ} \mathrm{C}$. The average and deviation of the hardness was $25 / \mathrm{HV} 0.3$ and less than $\pm 2.8 \%$, respectively.

Silicone KE1417 made by Shin-Etsu Chemical Ltd. (Tokyo, Japan) was used as a pressure medium, and a $3.5 \mathrm{wt} . \%$ curing agent was mixed with the liquid state silicone to make a semi-cylindrical shape with a diameter $D G=33.5 \mathrm{~mm}$ and a length $L G=170 \mathrm{~mm}$. The length of the semi-cylindrical rubber was sufficient so the end of the rubber did not reach the die opening when compressed by the punch. The semi-cylindrical rubber was used to save the rubber by the use of a semi-cylindrical sealing die, as shown in Figure 1. Naturally, cylindrical rubber can be used, as well. Due to the solid pressure medium, leakage of rubber from the cutout and the slit did not occur except at the opening of the die. The sealing was very easy. Figure 3 shows the results of the uniaxial compression test for two cylindrical silicone rubbers, N1 and N2, which are different production lots. Since rubber often is treated as an incompressible material [29], the strain on the rubber in the proposed rubber bulging test may be minimal when estimating it roughly from the compression ratio of it in the vicinity of the die opening. Moreover, the flow stress on the rubber is less than that on the tube metal. Thus, we assessed that the difference between rubber lots can be ignored.

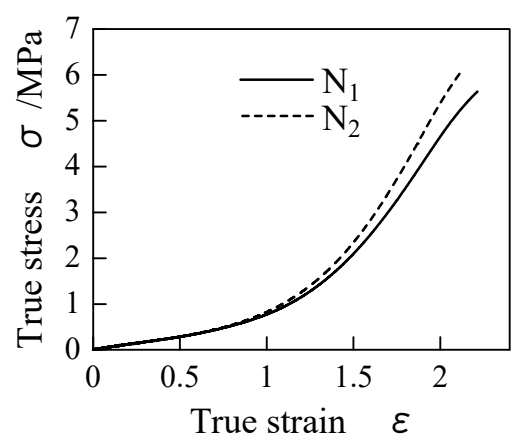

Figure 3. Stress-strain curve of rubber.

Figure 4 shows the used separate die. The hole of $\phi 38.2 \mathrm{~mm}$, which contacts the specimen, is dug to a T-shape by a milling machine with a ball-end mill. The corner roundness on the separated surface is $R=5 \mathrm{~mm}$, so the material moves to the opening easily. Although the ends of the tube can move in the rotation axis direction, the lower tube end is fixed to affect the asymmetrical deformation at the opening due to the tube rotation.

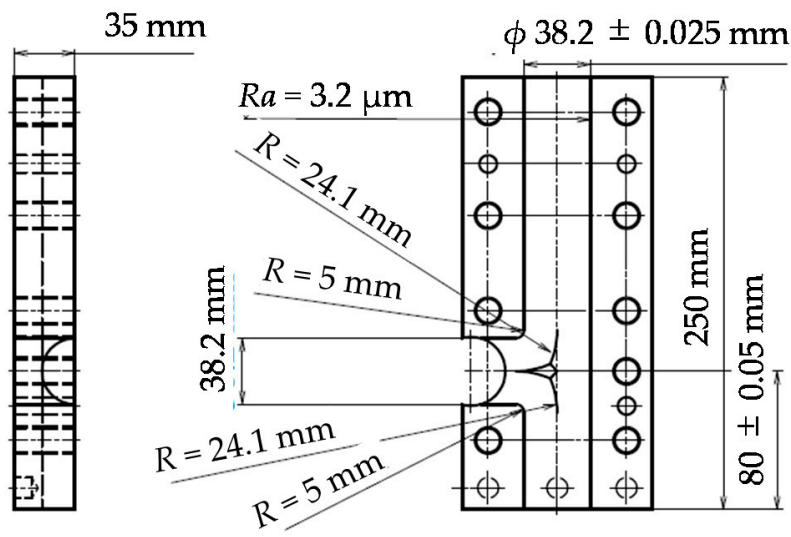

Figure 4. Dimensions of separate die (container). 


\subsection{The Test Procedure}

Although the used pure aluminum tubes seem quite homogeneous on the thickness and hardness distributions in comparison with the welded steel tube, the distributions could not be ignored due to the manufacturing process of hot porthole extrusion. Actually, even after the annealing treatment, the A1070TD specimens used have the effect of four joints in the circumferential direction of the deformation behavior. It was confirmed that the deformation behavior differs between the joint region and others by Matsuoka et al. [1,2]. Since it is difficult to exclude the locations that are easily fractured in tube forming, it is necessary to selectively evaluate the weak location. Regarding the reproducibility of the proposed test method, the fracture point must be at the center of the opening in all tests.

The procedure of the proposed test method is shown in Figures 5 and 6. Since tubes often have a weak position in the circumferential direction due to its production method, we realistically must check the forming limit of the weak position. Thus, we conducted the preliminary test to specify the weak position of the tube specimen. During the main test, the weak position was set at the center of the die opening.

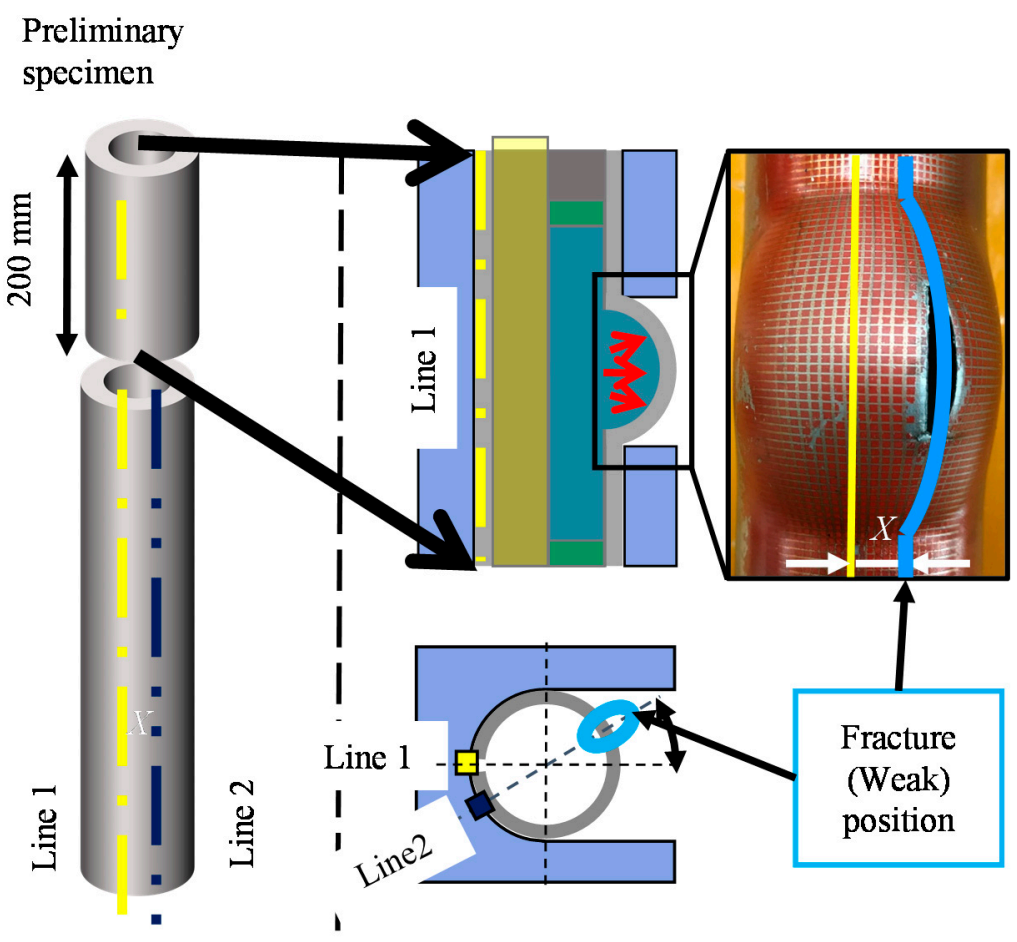

Figure 5. Overview of the preliminary test to determine the weak position.

Prior to cutting out the test material from the delivered $4000 \mathrm{~mm}$ tube, one marking line (line 1) that was parallel to the tube axial direction was drawn on the long pipe using a scriber. A specimen was cut out and grid lines were drawn at $180^{\circ}$ on the opposite side of line 1 to measure the circumferential position of the fracture. The specimen length was $200 \mathrm{~mm}$, and the grid lines were given using a laser-processing machine and painting. The preliminary test specimen was placed into a die to set line 1 in the $180^{\circ}$ opposite position of the opening. Then, a local rubber pressure one-sided bulge test was conducted. Shown in Figure 5, we regarded the circumferential position of the fracture as a weak position, and the $180^{\circ}$ opposite side position to the weak position as the position of Line 2. Axial marking line 2 is drawn at the $180^{\circ}$ opposite side of this weak point on the residual long tube. Shown in Figure 6, the main test specimen of the $200 \mathrm{~mm}$ tube was cut out from the residual tube, and line 2 was set to $180^{\circ}$ on the opposite side of the center of the opening of the die. Using this preliminary test method, the center of the bulged surface was fractured in all main tests, and the position always can be at the center of the opening, as captured 
by the imaging camera with a monocular lens. The reproducibility of the proposed test method was confirmed.

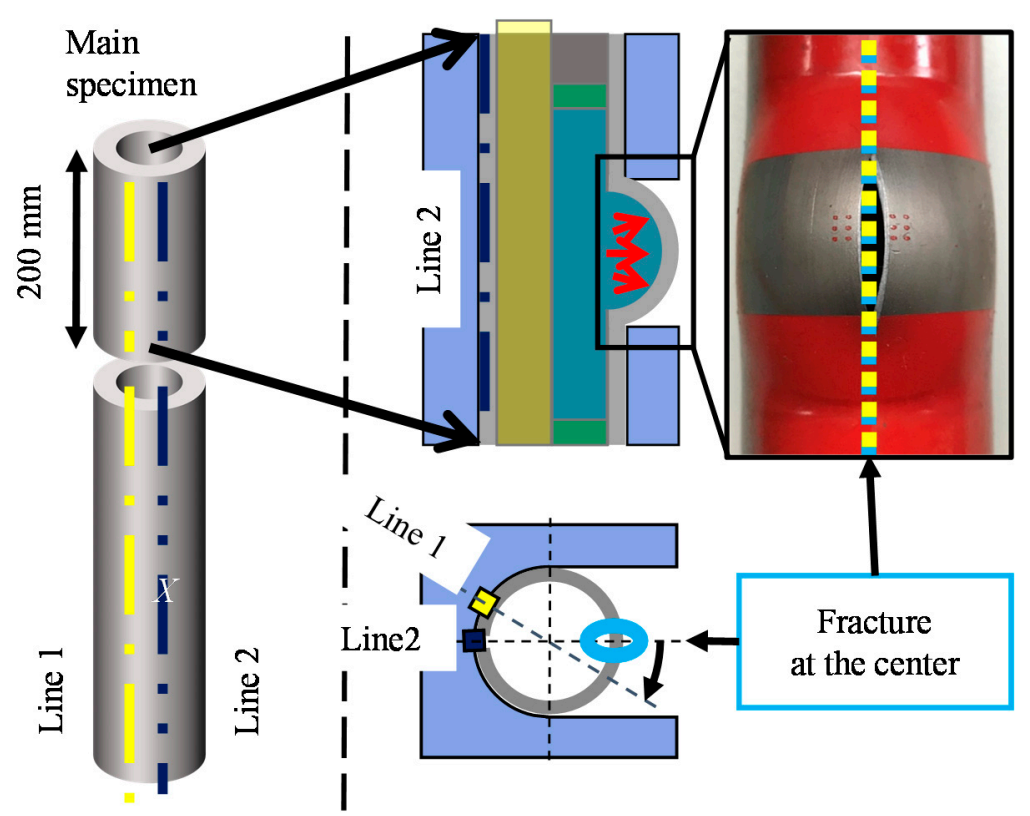

Figure 6. Overview of the main test.

To reduce friction over the contact surfaces of the sealing tool and silicone rubber with the test specimen, machine oil containing molybdenum disulfide was applied as a lubricant. The silicone rubber was inserted into the tube, and then urethane rubber for sealing was pushed from the upper side of it by a punch. Autograph AG-IS50kN (Shimadzu Corp., Kyoto, Japan) was used as a universal testing machine to push the punch, and the punch feed speed was $10 \mathrm{~mm} / \mathrm{min}$. The possibility to obtain several biaxial strain paths using the proposed testing method is examined in this paper.

\subsection{The Measurement Method of Strain Path by Image Processing with a Monocular Camera}

Prior to the main test, twelve red marking points for image processing were added to the specimens at the center of the die opening, as shown in Figure 7. During this research, red resin was coated over the pipe surface using a spray and the region, except for the marking points, was etched using a laser-processing machine. The strain path was acquired by measuring the centroid of each marking point using image processing. The attached red round points were $0.8 \mathrm{~mm}$ in diameter, and 12 points were arranged at $1.2 \mathrm{~mm}$ intervals. The camera was a NI-1776C smart camera (National Instrument Corp., Austin, TX, USA) with a monocular lens, and National Instruments LabVIEW (National Instrument Corp., Austin, TX, USA) was used as the recording software. The measurement data interval was 8 frames/s. The camera was placed vertical to the test material for the measurement, so the object distance between the lens and the test material was about $800 \mathrm{~mm}$, as shown in Figure 1. NI Vision Assistant was used as the image processing software (National Instrument Corp., Austin, TX, USA), and it calculated each centroid of each frame. Using this method, we obtained strain paths by determining the tube axial and circumferential strains. To obtain a forming limit at the occurrence of necking, strain components were measured near the fracture position, as shown in Figure 8. Although the deformed surface was curved, the accuracy, even by a monocular camera, was sufficient because the measured region at the center of the opening was small and vertical to the shooting axis. 


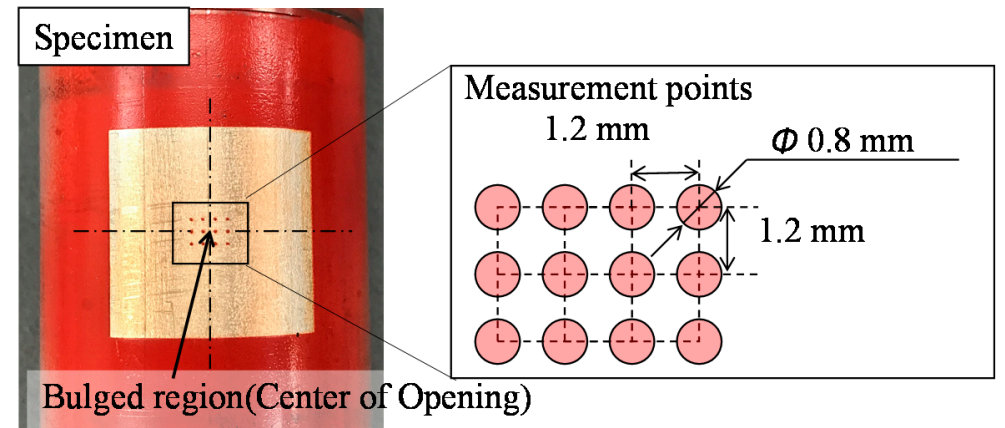

Figure 7. Size and shape of marking points for measurement by image processing.

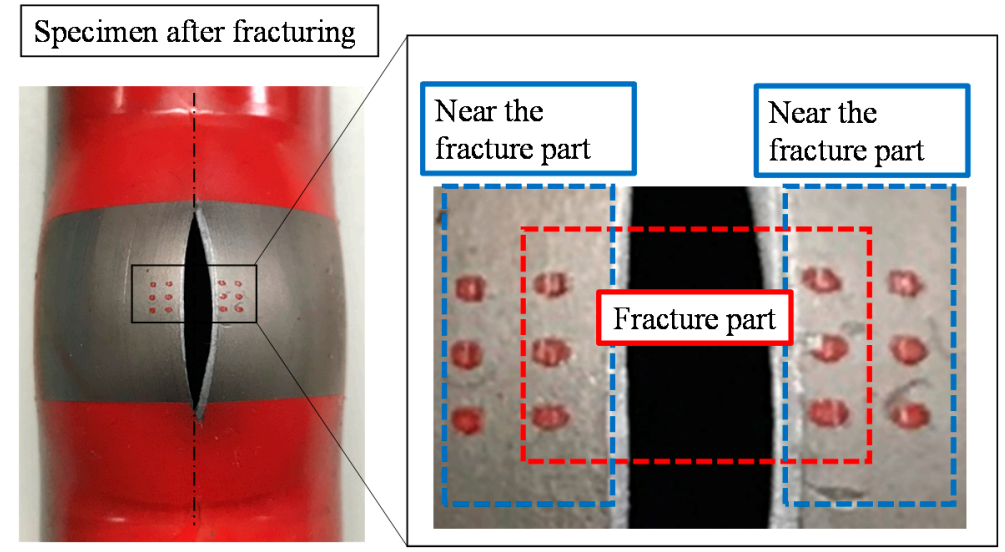

Figure 8. Appearance of measurement points on deformed tube.

\subsection{Cutout and Slit to Change a Strain Path}

Previously mentioned, it is supposed that cutouts and slits added to the specimen change the strain path. Thus, we examined the effect of cutout and slit shapes on the strain paths. The cutout shapes, rectangular, I-type, and round, shown in Figures 9 and 10, were examined. Cutout and slit were easily made with a general machine such as a milling machine or an electric discharge machine. Since cracks from the periphery, or corners of them, did not occur, there was no damage to the processed surface roughness, the roundness of the cutout corners, or the slit edges.

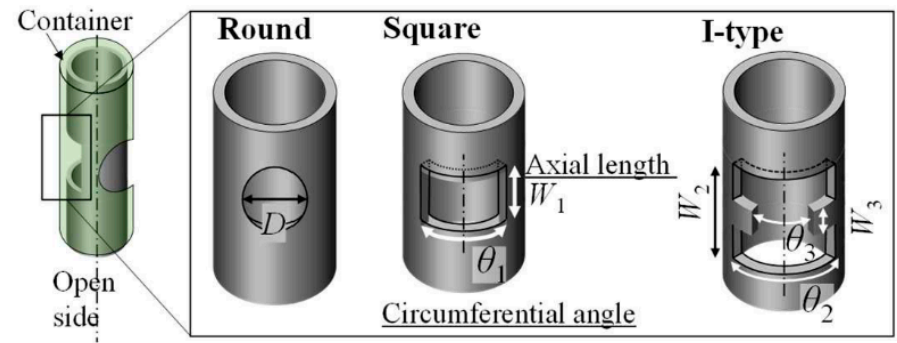

Figure 9. Variations of cutouts shape and position.

The cutout was made on the opposite side of the die opening, as shown in Figure 9. Regarding the case of the round cutout, the diameters $D$ were 10 and $30 \mathrm{~mm}$. Regarding the case of the rectangular cutout, the angle $\theta_{1}$ in the circumferential direction and the length $W_{1}$ in the axial direction were changed between $\theta_{1}=60-140^{\circ}$ and $W_{1}=10-60 \mathrm{~mm}$, respectively. The size of the cutout was limited because the deformed cutout ends reached the die opening during the test and the rubber might have leaked from the opening. Therefore, an I-type cutout was designed to prevent this leak of rubber by keeping the material near the opening. Concerning this paper, only one pattern of I-type was examined, 
and the cutout dimensions were $\theta_{2}=170^{\circ}, \theta_{3}=100^{\circ}$ and the lengths were $W_{2}=40 \mathrm{~mm}$ and $W_{3}=20 \mathrm{~mm}$, respectively. The round cutout was opened with a drill and its diameter was changed from 10 to $30 \mathrm{~mm}$. Shown in Figure 10, the slit was made in the specimen at the opening side at the $\pm 24 \mathrm{~mm}$ tube axial distant positions from the center of the opening. The length of the slit, $\theta_{4}$, also changed from 90 to $180^{\circ}$ in the circumferential direction. The wire diameter of the electric discharge machine was $0.2 \mathrm{~mm}$.

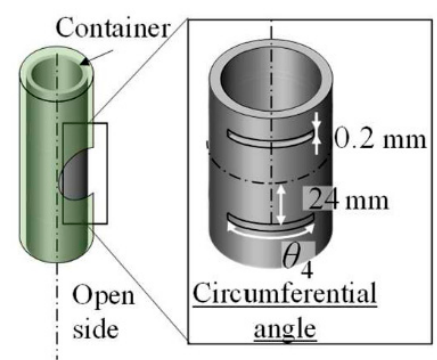

Figure 10. Size and position of slits.

\section{Results and Discussion}

\subsection{Measuring Precision of Strain by a Monocular Camera}

We obtained strain paths of the specimen using the proposed method. Figure 11 shows the strain path of a specimen with no cutout, and a forming limit strain measured by a microscope. Comparing the final strain on the strain path using image processing and the forming limit strain measured by a microscope, the average discrepancy of the circumferential strain is 0.026 , and that of the axial strain is 0.020 . It is considered that the discrepancy of $6-8 \%$ was a calculation error of the image processing caused by the marker' peeling or deforming after the necking. Actually, the value measured by a microscope was on the strain path obtained using image processing. Thus, we regarded that the proposed method, using image processing, could be used as a strain measurement method. Moreover, we determined the object distance was $800 \mathrm{~mm}$ and the marker distance and the measurement error were about several percent different due to the pixel number of the camera. The object distance, however, could be 250-300 $\mathrm{mm}$ if the diaphragm of the lens used was larger using a defused lighting method. It is estimated that a less than $0.3 \%$ error can be achieved by this camera.

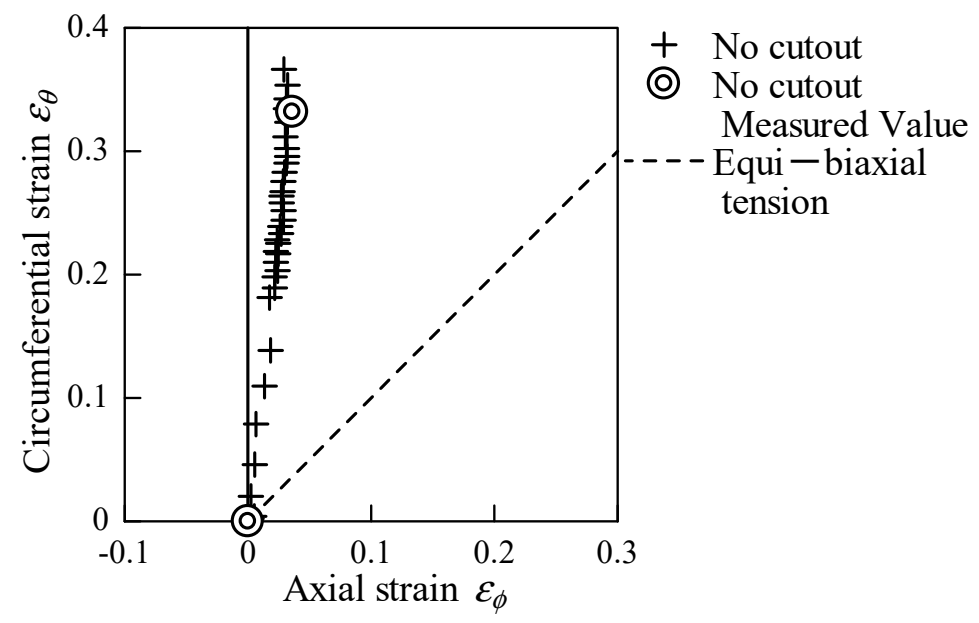

Figure 11. Strain path measured by image processing and forming limit strain measured by microscope.

Next, we verified whether the obtained strain paths were proportional loading states or not. Concerning this paper, the strain ratio is defined as the axial strain divided by the circumferential strain at the necking, which is measured near the fracture. The linear approximation of each strain path was conducted. The average value of the $R^{2}$ representing 
the errors were 0.95 for no cutout, 0.92 for cutout, and 0.89 for slit. Therefore, we can confirm that the obtained strain paths are almost close to the proportional loading state. Different from hydraulic bulging, the inner pressure distribution for rubber bulging may be nonuniform. Flow stress in the porthole extrusion tube is inhomogeneous. We have not established the numerical analysis model yet due to the difficulty of their treatments, and the reason for this proportional loading state deformation could not be clarified.

\subsection{Cutout and Slit}

During the tube bulge hydroforming, the strain path is often between circumferential uniaxial tension and equal biaxial tension, and it covers the strain ratio between -0.5 and 1.0. Shown in Figure 11, the strain ratio was 0.080 for the specimen without the cutout. Figure 12 shows the results for round cutouts. The strain ratios of $D=10 \mathrm{~mm}$ and $30 \mathrm{~mm}$ are 0.18 and 0.24 , respectively. When the round cutout diameter $D$ is larger, the closer the strain paths are to the equi-biaxial tension. However, even for a cutout diameter of $D=30 \mathrm{~mm}$, the strain ratio is small. The residual material around the die's opening abounds and it is considered that the effect of the cutout on the material flow to the opening makes it smaller.

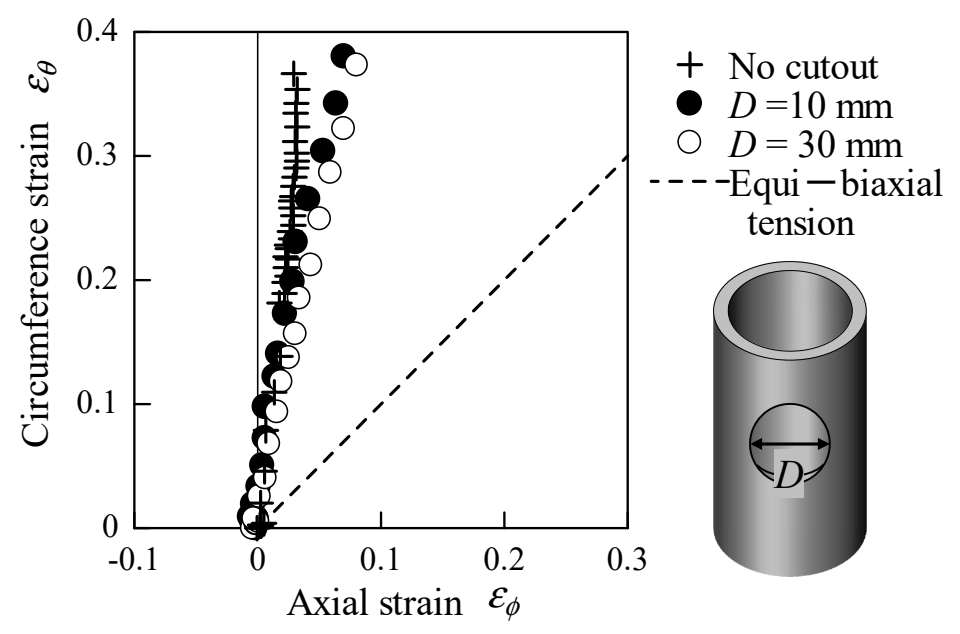

Figure 12. Strain paths obtained by specimens with round cutouts.

Figure 13 shows the strain paths of specimens with square cutouts. Figure 13a shows the change in length $W$ at the circumferential angle of $\theta_{1}=140^{\circ}$; when $W_{1}=10,30$, and $60 \mathrm{~mm}$, the strain ratios are $0.13,0.21$, and 0.48 , respectively. Thus, the larger $W_{1}$ is, the closer the strain paths are to the equi-biaxial tension. Viewing Figure $13 \mathrm{~b}$, the length $W_{1}=30 \mathrm{~mm}$ and the angle $\theta_{1}$ in the circumferential direction is changed. Occurring at $\theta_{1}=60,140^{\circ}$, the strain ratios are 0.11 and 0.21 , respectively. It was found that the larger $\theta_{1}$ was, the closer it was to the equi-biaxial tension, as well. Therefore, it is supposed that as the cutout area becomes larger, the material around the cutout easily moves to the die's opening in the circumferential direction, as shown in Figure 2 and, as a result, the effect of the circumferential deformation is smaller than that of the tube axial.

Figure 14 shows the strain paths of specimens with an I-type cutout. Although the square cutout of more than approximately $\theta_{1}=140^{\circ}$ tends to cause rubber leakage, the cutout area can increase at the corner in the circumferential direction without rubber leakage using the I-type cutout. Considering the case of $\theta_{2}=170^{\circ}$, the strain ratio was 0.67 , which was larger than the 0.48 obtained by a square cutout of $\theta_{1}=140^{\circ}$. It is expected that the strain ratio can be closer to the equi-biaxial tension via optimization of the cutout shape, for example, $\theta_{3}$ or $W_{3}$. 


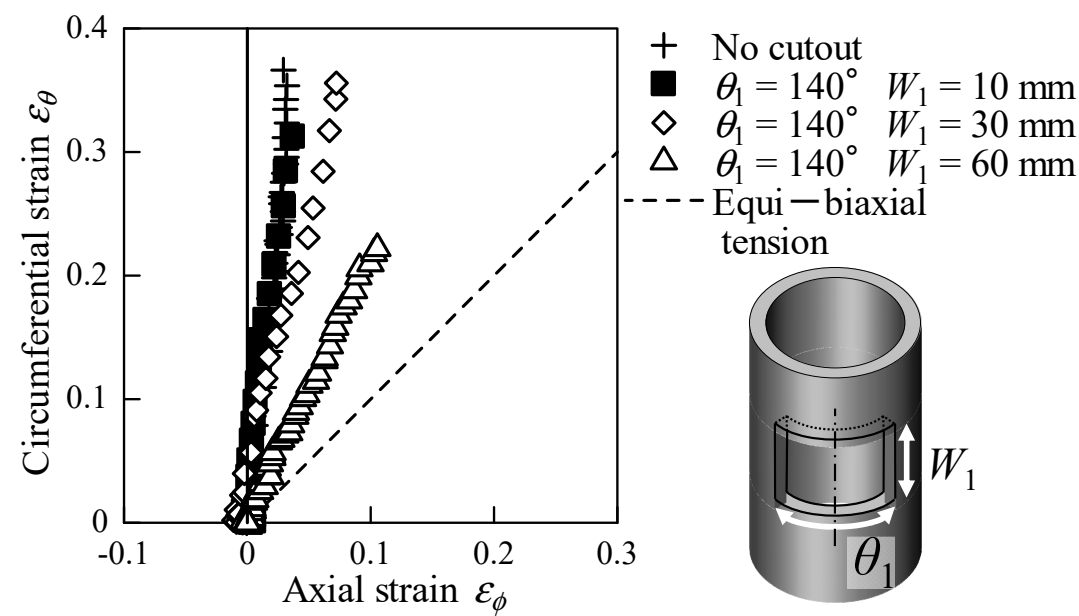

(a)

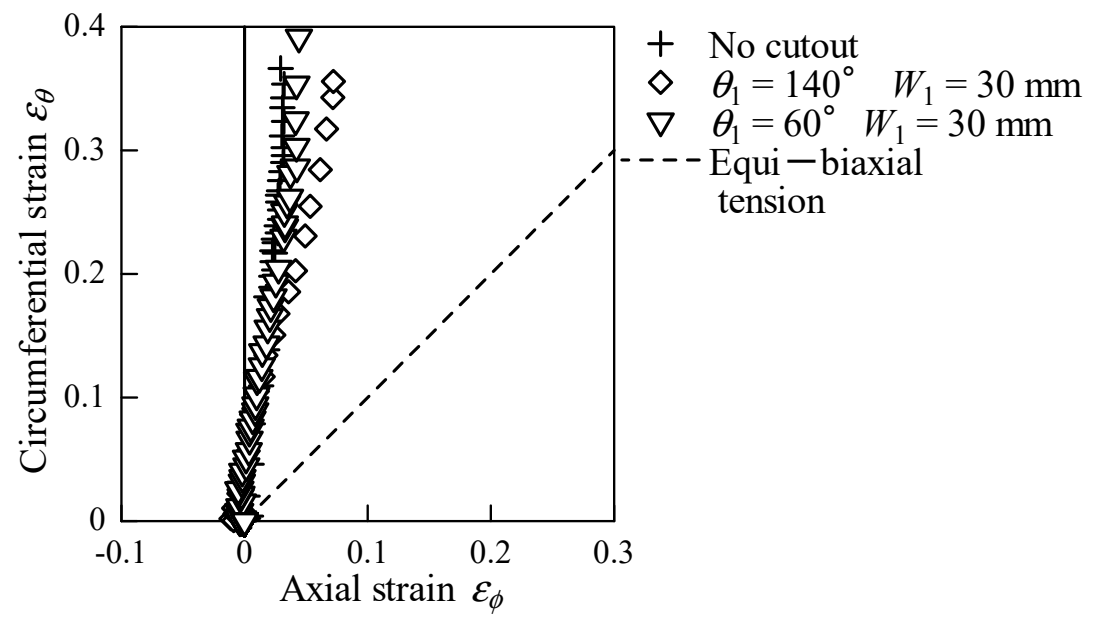

(b)

Figure 13. Strain paths of specimen with and without a square cutout; (a) different axial length $W_{1}$ and circumferential angle $\theta_{1}$ of $140^{\circ}$ (b) different circumferential angle $\theta_{1}$ and axial length $W_{1}$ of $30 \mathrm{~mm}$.

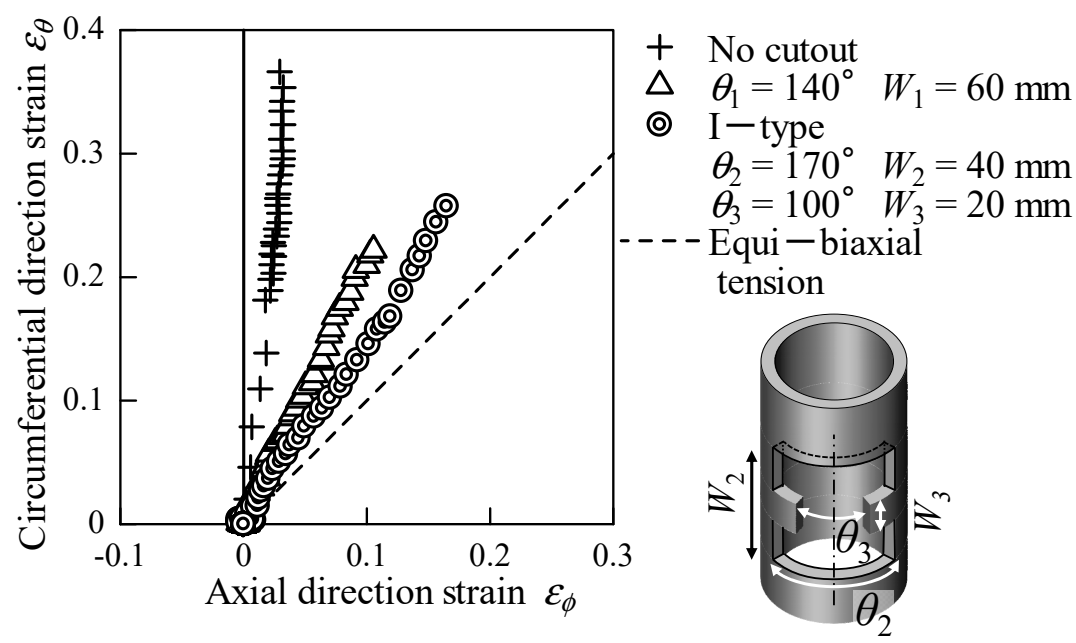

Figure 14. Strain paths of specimen with an I-type cutout.

Figure 15 shows the strain paths of specimens with slits. The strain ratios defined by this paper for slit sizes of $\theta_{4}=90^{\circ}, 120^{\circ}$, and $150^{\circ}$ are $-0.12,-0.11$, and -0.14 , respectively. 
Via giving two slits on the tube, the strain ratio is closer to the uniaxial tension's value, -0.5 , in comparison with the specimen without a cutout and slit. The material near the slit seems to be moved in the tube axial direction, as shown in Figure 2. Although the larger $\theta_{4}$ is, the closer the strain paths look to uniaxial tension, the change is very small. Although the region between the two slits is circumferentially elongated like the uniaxial tension test, the circumferential ends of the region are connected to the tube. The distance between the two slits is $48 \mathrm{~mm}$ and the circumferential length of the region is short, even if $\theta_{4}=150^{\circ}$. Therefore, the circumferential ends of the region are restricted to not being shrunk in the tube axial direction. Consequently, it is thought that the effect of the slit length on the strain path is slight.

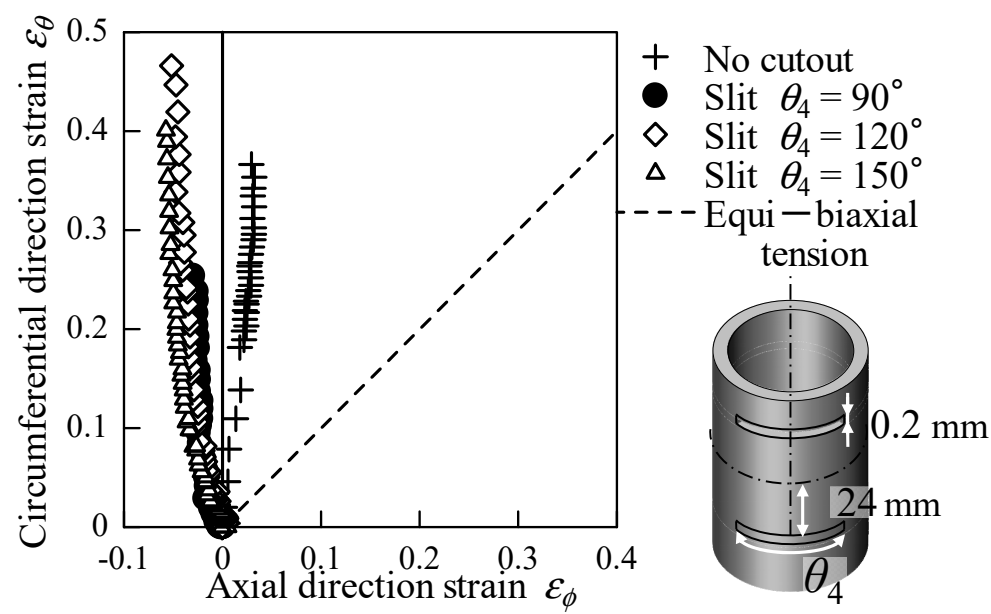

Figure 15. Strain paths of specimen with slits.

Regarding cases of a cutout, the end of each strain path is different from the biaxial test results of the metal sheet, as well. Concerning this paper, the strain ratio is defined by an average of the strain calculated from the distance between two markers, except for the fractured position. The distance is subjected to the effect of the local circumferential elongation at the fracture and it is guessed that despite the near stain path, the periods of the strain path end may be quite different. Evidently, although this phenomenon occurs in the case of the metal sheet, the tubes produced using porthole extrusion have a strong inhomogeneity in the circumferential direction and the deviation of the strain on both sides of the fracture is very large. To obtain the forming limit for the metal tubes, it is desired that the deformation behavior, and how to decide the forming limit, will be clarified by continuous research using a 3-D DIC system.

\section{Conclusions}

A local one-sided rubber bulging testing method was proposed to measure proportional strain paths in the right and left side of the strain space. To obtain the various strain paths in the biaxial deformation of the tube axial and circumferential directions, a cutout was preprocessed in the tubal specimen at the opposite side of the bulged surface, and slits also were made beforehand in the vicinity of the bulged surface in the tube axial direction. The size and shape of them were changed and we examined the effect of these on the strain paths. The testing method was applied to an annealed pure aluminum tube of A1070TD, and we confirmed that all measured strain paths almost were proportional. Logically, using the proposed testing method, some strain paths with strain ratios from -0.14 to 0.67 were obtained using tubular specimens. A larger cutout resulted in a strain path that was closer to the equi-biaxial strain path, and a larger slit resulted in a strain path that was closer to the uniaxial strain path. Although, at present, the forming limit on each strain path is roughly specified from imaging processing, the specification method is the next research stage. 


\begin{abstract}
Author Contributions: Conceptualization, H.Y.; methodology, H.Y.; validation, H.Y., K.N. and M.O.; formal analysis, K.N.; investigation, H.Y. and K.N.; resources, H.Y. and M.O.; data curation, K.N.; writing-original draft preparation, K.N.; writing-review and editing, H.Y. and M.O.; visualization, K.N.; supervision, H.Y.; project administration, H.Y.; funding acquisition, H.Y. All authors have read and agreed to the published version of the manuscript.
\end{abstract}

Funding: This research received no external funding.

Conflicts of Interest: The authors declare no conflict of interest.

\title{
References
}

1. Matsuoka, H.; Neumann, L.; Hamano, H.; Sakaguchi, M. Effect of microstructures on tensile properties of a 6061 extruded alloy containing weld parts. J. Jpn. Inst. Light Met. 2002, 52, 155-160. (In Japanese) [CrossRef]

2. Eda, H.; Sakaguchi, M.; Maehara, H.; Sukimoto, M.; Fuchizawa, S. Effects of production conditions on free bulge formability of 6063 aluminum alloy tubes. J. Jpn. Inst. Light Met. 1993, 43, 438-443. (In Japanese) [CrossRef]

3. Yoshida, Y. Tensile test and evaluation tests of tube and pipe. J. Jpn. Soc. Technol. Plast. 2010, 51, 313-317. (In Japanese) [CrossRef]

4. Yoshida, Y.; Kume, S.; Yukawa, N.; Ishikawa, T.; Manabe, K. Tube Tension Test Using Image Analysis. In Proceedings of the 148th the Iron and Steel Institute of Japan Meeting, 17-5, Akita, Japan, 28-30 September 2004; p. 1054. (In Japanese)

5. Yoshida, Y.; Yukawa, N.; Ishikawa, T. Evaluation of Fracture Property of Tube by Tensile Test Using Image Analysis. In Proceedings of the 4th International Conference on Tube Hydroforming (Tubehydro 2009), Kaohsiung, Taiwan, 6-9 September 2009; pp. 73-78.

6. Yoshimura, H.; Mihara, Y. Applicability of Mechanical Property of Steel Tubes by Ring Tensile Test. In Proceedings of the 166th the Iron and Steel Institute of Japan Meeting, 26-2, Kanazawa, Japan, 17-19 September 2013; pp. 611-614. (In Japanese)

7. Yoshimura, H.; Tajima, M.; Mihara, Y. Estimation of Ductile Fracture of Tube Material by Ring Tensile Test. In Proceedings of the 5th International Conference on Tube Hydroforming (Tubehydro 2011), Noboribetsu, Japan, 24-27 July 2011 ; pp. $203-206$.

8. Sokolowski, T.; Gerke, K.; Ahmetoglu, M.; Altan, T. Evaluation of tube formability and material characteristics: Hydraulic bulge testing of tubes. J. Mater. Process. Technol. 2000, 98, 34-40. [CrossRef]

9. Koç, M.; Aue-U-Lan, Y.; Altan, T. On the characteristics of tubular materials for hydroforming-Experimentation and analysis. Int. J. Mach. Tools Manuf. 2001, 41, 761-772. [CrossRef]

10. Hwang, Y.M.; Lin, Y.K.; Altan, T. Evaluation of tubular materials by a hydraulic bulge test. Int. J. Mach. Tools Manuf. 2007, 47, 343-351. [CrossRef]

11. Manabe, K.; Yoshida, Y. Effect of Lubricants in the Flaring Test of Steel Tubes. In Proceedings of the 148th the Iron and Steel Institute of Japan Meeting, 17-5, Akita, Japan, 28-30 September 2004; p. 1053. (In Japanese)

12. Manabe, K.; Nakata, K. 2736 Effect of Tube-end Finishing Process on Deformability of Steel Tubes by Flaring Test. In Proceedings of the Mechanical Engineering Congress (MECJ-07), Osaka, Japan, 9-12 September 2007; pp. 633-634. [CrossRef]

13. Oyane, M. On criteria for ductile fracture (Minor special issue on plastic working and materials characteristics). J. Jpn. Soc. Mech. Eng. 1972, 75, 596-601. (In Japanese) [CrossRef]

14. Manabe, K.; Yoshida, Y. Evaluation of Hydroformability of Steel Pipes by Conical Flaring Test. In Proceedings of the 3rd International Conference on Tube Hydroforming (Tubehydro 2007), Harbin, China, 14-17 November 2007; pp. 39-45.

15. Guo, X.; Ma, F.; Guo, Q.; Luo, X.; Kim, N.; Jin, K. A calculating method of tube constants of ductile fracture criteria in tube free bulging process based on M-K theory. Int. J. Mech. Sci. 2017, 128-129, 140-146. [CrossRef]

16. Hwang, Y.M.; Lin, Y.K.; Chuang, H.C. Forming limit diagrams of tubular materials by bulge tests. J. Mater. Process. Technol. 2009, 209, 5024-5034. [CrossRef]

17. Kim, J.; Kim, S.W.; Song, W.J.; Kang, B.S. Analytical and numerical approach to prediction of forming limit in tube hydroforming. Int. J. Mech. Sci. 2005, 47, 1023-1037. [CrossRef]

18. Song, W.J.; Heo, S.C.; Ku, T.W.; Kim, J.; Kang, B.S. Evaluation of effect of flow stress characteristics of tubular material on forming limit in tube hydroforming process. Int. J. Mach. Tools Manuf. 2010, 50, 753-764. [CrossRef]

19. Kuwabara, T.; Moriguchi, K. Formability Evaluation of Steel Tubes Using a Simplified Hydraulic Bulging Test. In Proceedings of the 148th the Iron and Steel Institute of Japan Meeting, 17-5, Akita, Japan, 28-30 September 2004; p. 1051. (In Japanese)

20. Yoshida, K.; Kuwabara, T.; Takahashi, S. Stress Based Forming Limit Criterion for Aluminum Alloy Tubes. In Proceedings of the 2003 Annual Meeting of the JSME/MMD (M\&M2003), Toyama, Japan, 24-26 September 2003; pp. 821-822. (In Japanese) [CrossRef]

21. Sugawara, F.; Kuwabara, T. Development of a multiaxial tube expansion testing machine that enables the continuous measurement of large-strain biaxial stress-strain curves of sheet metals. J. Jpn. Soc. Technol. Plast. 2013, 54, 57-63. (In Japanese) [CrossRef]

22. Korkolis, Y.P.; Kyriakides, S. Inflation and burst of anisotropic aluminum tubes for hydroforming applications. Int. J. Plast. 2008, 24, 509-543. [CrossRef]

23. Shirayori, A. Effect of Circumferential Initial Imperfection on Hydraulic Bulging of ERW Steel Tubes. In Proceedings of the 5th International Conference on Tube Hydroforming (Tubehydro 2011), Noboribetsu, Japan, 24-27 July 2011; pp. 179-182.

24. Shirayori, A.; Oshima, R.; Narazaki, M. Effect of Axial Feeding in Partial Hydraulic Bulging of ERW steel tube. In Proceedings of the 62nd Japanese Joint Conference for the Technology of Plasticity, Toyohashi, Japan, 26-29 October 2011; pp. 165-166. (In Japanese) 
25. Chen, X.; Yu, Z.; Hou, B.; Li, S.; Lin, Z. A theoretical and experimental study on forming limit diagram for a seamed tube hydroforming. J. Mater. Process. Technol. 2011, 211, 2012-2021. [CrossRef]

26. Li, S.; Chen, X.; Kong, Q.; Yu, Z.; Lin, Z. Study on formability of tube hydroforming through elliptical die inserts. J. Mater. Process. Technol. 2012, 212, 1916-1924. [CrossRef]

27. Magrinho, J.P.; Silva, M.B.; Centeno, G.; Moedas, F.; Vallellano, C.; Martins, P.A.F. On the determination of forming limits in thin-walled tubes. Int. J. Mech. Sci. 2019, 155, 381-391. [CrossRef]

28. Magrinho, J.P.; Centeno, G.; Silva, M.B.; Vallellano, C.; Martins, P.A.F. On the formability limits of thin-walled tube inversion using different die fillet radii. Thin-Walled Struct. 2019, 144, 106328. [CrossRef]

29. Gent, A.N. A new constitutive relation for rubber. Rubber Chem. Technol. 1996, 69, 59-61. [CrossRef] 